\title{
Relevância e influência dos Stakeholders dos Hospitais Filantrópicos e Religiosos
}

\author{
Relevance and Influence of the Stakeholders in Religious and \\ Philanthropic Hospitals
}

Fábio Augusto Martins ${ }^{1}$; Amélia Cristina Ferreira-da-Silva ${ }^{2}$; Carlos Machado-Santos ${ }^{3}$

1 E-mail: famart30@hotmail.com; Universidade Trás-os-Montes e Alto Douro; Centro Universitário do Planalto de Araxá - UNIARAXÁ

$2 \quad$ E-mail: $\quad$ ameliafs@iscap.ipp.pt; Institute of Accounting and Administration of Porto; Centro de Estudos em Economia, População e Sociedade - CEPESE, CECEJ

E-mail: $\quad$ cmsantos@utad.pt; Universidade de Trás-os-Montes e Alto Douro; Centro de Estudos em Economia, População e Sociedade CEPESE

\begin{abstract}
Resumo
Partindo da premissa de que o gerenciamento dos stakeholders é fundamental na estratégia das organizações, este artigo tem como objetivo captar a perceção dos trabalhadores dos hospitais filantrópicos religiosos sobre a importância relativa e influência dos diversos stakeholders. Os dados foram recolhidos através de um questionário distribuído nos hospitais de Araxá, MG. Os resultados evidenciaram os médicos, os pacientes e o Sistema Único de Saúde como as principais partes interessadas. Tratando- se de instituições filantrópicas, altamente dependentes de legitimação social e financeiramente frágeis, surpreende a pouca importância reconhecida aos doadores financeiros. Além de proporcionar a identificação dos stakeholders deste tipo específico de organização hospitalar, este artigo explora oportunidades e desafios que se colocam à gestão da relação do hospital com estas partes interessadas.

Palavras-chave: Stakeholders; Hospitais filantrópicos; Organização estratégica.
\end{abstract}

\begin{abstract}
Assuming that the management of stakeholders is key in the strategy of organizations, this article aims to capture the perception of workers in religious charitable hospitals regarding the relative importance and influence of various stakeholders. Data was collected through a questionnaire distributed in hospitals in Araxa, Brazil. The results showed that doctors, patients and the National Health System were considered to be key stakeholders. Considering that these organizations are charities and, thus, financially fragile, and highly dependent on social legitimacy, the low priority accorded to financial donors was surprising. In addition to identifying the stakeholders of this specific type of hospital organization, this article explores the opportunities and challenges faced by hospital managers in their relationship with these interested parties.
\end{abstract}

Keywords: Stakeholders; Philanthropic hospitals; Strategic organization.

\section{Introdução}

A gestão dos stakeholders é fundamental para a estratégia organizacional. Uma vasta literatura sobre o assunto apresenta a identificação das partes interessadas como uma etapa fundamental do processo de gestão. Como os hospitais são organizações complexas, muitos são os seus interessados. Os hospitais filantrópicos, dada a sua missão eminentemente social, são organizações particularmente complexas, pois, além dos desafios enfrentados pelas organizações hospitalares em geral, confrontam-se com a escassez de recursos próprios das organizações do terceiro setor. Assim, embora haja um amplo consenso sobre as 
partes interessadas dos hospitais em geral, é reconhecido na literatura que cada organização tem seu próprio mapa de stakeholders (Fassin, 2009; Freeman, 1984).

Esta pesquisa tem como objetivo identificar os atores de maior relevância nos hospitais filantrópicos, de origem religiosa, a partir da perspetiva de seus funcionários. Os resultados são representados e interpretados a partir de um gráfico que pretende traduzir a importância relativa e a relação de influência dos interessados com os hospitais.

Este trabalho assume a forma de uma pesquisa essencialmente descritiva. Os dados foram recolhidos através de um inquérito por questionário realizado com trabalhadores dos hospitais de Araxá (MG). Dos 380 questionários distribuídos, apenas 117 foram recebidos e considerados válidos.

Nas seções seguintes procuramos apresentar os fundamentos da teoria dos Stakeholders e evidenciar a sua relevância e aplicabilidade já demonstradas na investigação científica presente na literatura internacional. $\mathrm{O}$ método e os procedimentos metodológicos aplicados nesta pesquisa são descritos na secção III, à qual se segue a apresentação e discussão dos resultados. Considerando que nessa pesquisa utilizamos uma amostra pouco representativa, qualquer generalização exige cuidado. Não obstante, embora os dados sejam insuficientes para obter uma matriz completa, cremos que ainda assim o trabalho fornece novos esclarecimentos sobre quem são os grupos de partes interessadas nos hospitais filantrópicos de origem religiosa.

\section{Enquadramento Teórico da Questão de Investigação}

A análise das partes interessadas se desenvolveu como uma ferramenta ou conjunto de ferramentas, com diferentes propósitos em sua aplicação nas áreas de política, gestão e implementação de projetos (Brugha \& Varvasovszky, 2000). A introdução da perspetiva dos stakeholders, no âmbito da gestão estratégica, surgiu em meados da década de 1980 - com a publicação da obra A Stakeholder Approach to Strategic Management, por Freeman (1984) - e tem proporcionado um vasto campo de investigação em gestão. A análise das partes interessadas é agora sem dúvida mais importante do que nunca, devido à natureza do mundo cada vez mais interligado (Bryson, 2004). Portanto, o envolvimento dos atores se tornou um tema de grande atenção, tanto na literatura como na prática organizacional (Achterkamp \& Vos, 2007). A teoria dos Stakeholders afirma que as empresas têm uma responsabilidade social de considerar os interesses de todos os atores afetados por suas decisões de negócios (Avetisyan \& Ferrary, 2013). De forma indireta, segundo Tullberg (2013), quase todo mundo pode, realmente, ser visto como afetados por uma organização em algum grau. Mas, se não houver uma contribuição concreta ou se não tiver um papel específico na organização, isso não deve ser considerado suficiente para ser vista como parte interessada.

A elaboração estratégica estabelece como tarefa extremamente relevante, a gestão de interface entre as muitas exigências - às vezes concorrentes - dos diferentes stakeholders de uma organização em relação aos seus fins estratégicos (Ackermann \& Eden, 2011). Entretanto, como um problema de gestão, a identificação das partes interessadas não tem sido facilmente resolvida (Achterkamp \& Vos, 2007; Vos, 2003), especialmente nas organizações complexas continua a ser difícil de concretizar (E W Mainardes, 2010). Qualquer definição de uma parte interessada deve levar em conta as relações interessadosorganização (Avetisyan \& Ferrary, 2013). Friedman e Miles (2006) apresentaram um resumo de cinquenta e cinco definições de stakeholders com um campo de abrangência de setenta e cinco textos 
organizados em ordem cronológica. A definição mais antiga é, em muitas vezes, creditado a um memorando interno produzido em 1963 pelo Stanford Research Institute. Mas, a que é comumente considerada - pelo menos nos círculos acadêmicos, como texto seminal dos interessados - a definição clássica (Freeman, 1984), determina que um participante de uma empresa possa ser qualquer grupo ou indivíduo que possa afetar ou ser afetado pela realização dos objetivos organizacionais (Figura 1). As partes interessadas são aquelas que têm relações voluntárias ou involuntárias com a empresa e pode afetála dentro do quadro único de contrato implícito ou moral (Avetisyan \& Ferrary, 2013).

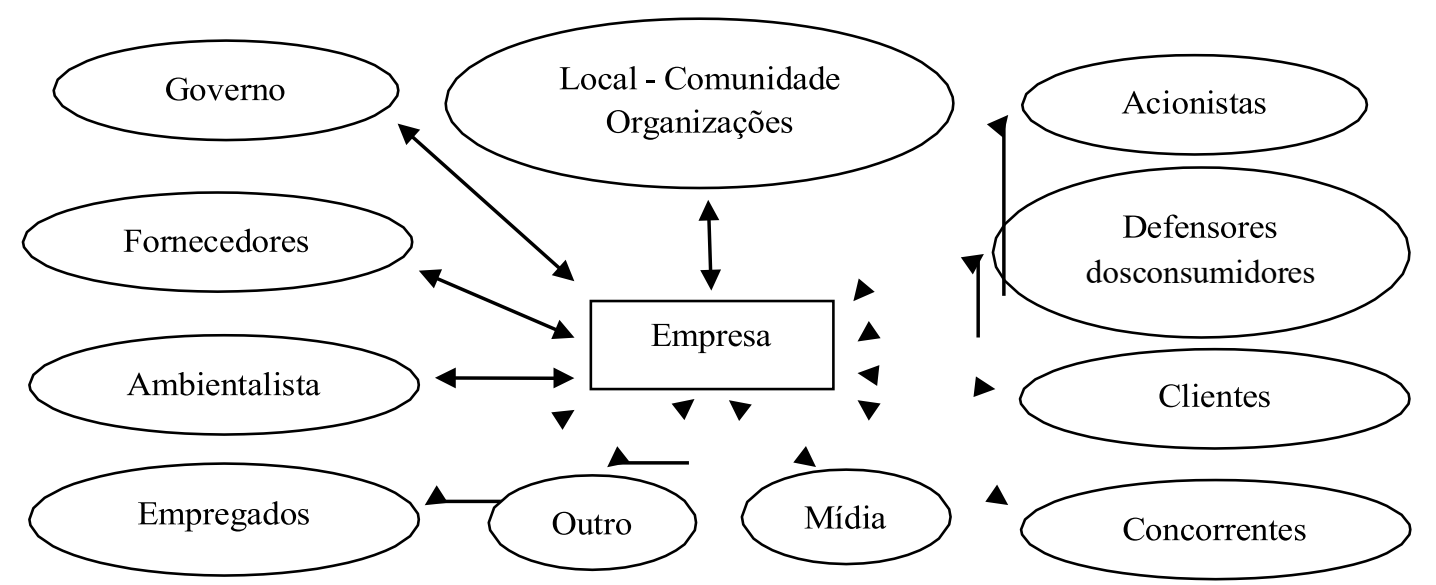

Figura 1. Modelo de participação adaptado de Freeman (1984, p.25)

Então, os stakeholders são muitos grupos e organizações de pessoas que estão envolvidas ou são afetadas ou que tenham alguma responsabilidade parcial para agir (Bryson, 2004), entretanto, aqueles que podem afetar uma organização nem sempre são os mesmos que podem ser afetados por ela (Fassin, 2009). Portanto, não há acordo sobre quem são os interessados na organização e a quem os gestores devem atenção (Mitchell, Agle, \& Wood, 1997). Por isso não é de esperar uma definição única, universalmente aceita (Kaler, 2002), do que é ser uma das partes interessadas em um negócio, ou seja, uma ideia razoavelmente clara do que se entende pelo termo "stakeholder" em relação às empresas. Mahoney (2012) defende que partes interessadas são aquelas que beneficiam ou podem beneficiar com a prosperidade da organização e sofrem ou podem sofrer perdas econômicas quando a organização atravessa problemas de sustentabilidade e/ou sobrevivência.

Mitchell, Agle, e Wood (1997), com o objetivo de definir o princípio de quem e o que realmente importa, deram rumo a uma teoria da identificação das partes interessadas que tem como base a ideia de que a relevância dos interessados pode ser aferida a partir da presença de um ou mais dos três atributos de relacionamento: poder dos interessados para influenciar a empresa, legitimidade do relacionamento do stakeholder com a organização e a urgência com que os interessados reivindicam a chamada de atenção imediata por parte do negócio. Portanto, Achterkamp e Vos (2007) argumentam que Mitchell e seus colaboradores focaram na questão de como os gestores escolhem suas partes interessadas e como eles priorizam entre as reivindicações dos interessados concorrentes. A ideia prática básica da teoria das partes interessadas, para Tullberg (2013), é que o sucesso de uma empresa é muito dependente de uma boa 
cooperação com as partes interessadas. Do que se segue o conselho para prestar muita atenção para as necessidades e desejos destes stakeholders.

Lafreniere, Deshpade, Bjornlund, e Huntera (2013) procuraram entender, através de investigação narrativa, como os atores percebem seus interesses em uma organização e, consequentemente, como decidem influenciá-la. Com isso investigaram a existência ou não de uma desconexão entre a perceção dos gestores de recursos naturais e dos stakeholders ao implementarem iniciativas de gestão de transferência de água na província de Alberta, no Canadá. Para isso utilizaram o modelo de Mitchell et al. (1997), como base para investigar os interessados por sua capacidade de se concentrar na identificação, na categorização e na priorização das reivindicações das partes interessadas.

Os interessados também podem interagir, cooperar e formar alianças com outras partes interessadas (um interessado pode persuadir outro mais poderoso para juntar-se a sua reclamação contra uma organização). Neville e Menguc (2006) desenvolveram uma estrutura para compreender e medir os efeitos sobre a organização de interações das partes interessadas concorrentes, complementares e cooperativas, o que eles referem como a multiplicidade das partes interessadas.

Bryson (2004) centra-se especificamente na análise de partes interessadas que possam ser úteis aos gestores públicos, seja para ajudar a sua organização a um melhor desempenho diretamente, ou para ajudar a criar um "ambiente de autorização" que, indiretamente, possa melhorar o desempenho da organização. Seu trabalho está organizado em torno da literatura de gestão estratégica orientada para o setor público.

A gestão de partes interessadas é uma parte essencial do gerenciamento de projetos (Aaltonen, Jaakko, \& Tuomas, 2008; Olander \& Landin, 2005). Uma atitude negativa pelas partes interessadas em um projeto de construção pode obstruir severamente a sua execução (Olander \& Landin, 2005). Aaltonen, Jaakko, e Tuomas (2008), combinaram o quadro de relevância das partes interessadas (Mitchell et al., 1997) com estratégias de influência dos stakeholders (Frooman, 1999) para identificar as estratégias que os atores tentam usar para aumentar a sua importância aos olhos do gerenciamento de projetos e, assim, influenciar o resultado, em projetos globais.

Parent e Deephouse (2007) examinaram a identificação dos interessados e a priorização pelos gestores com base no quadro teórico de Mitchell et al. (1997) - em um estudo de natureza exploratória, utilizando casos comparativos de dois comitês organizadores de eventos esportivos de grande escala, com um foco particular em entrevistas com gestores em três níveis hierárquicos. Concluíram que o nível hierárquico e o papel dos gestores têm efeitos diretos na moderação das partes interessadas sobre a identificação e a importância percebida e que dos três atributos de relacionamento, propostos por Mitchell e seus colaboradores, o poder tem o efeito mais importante sobre a relevância, seguido pela urgência e a legitimidade.

Os stakeholders em um negócio devem ter algum tipo de reclamação sobre os serviços dessa organização. Para definições desse tipo que muito inequivocamente importa é que os interessados possam influenciar o funcionamento do negócio de alguma forma (Kaler, 2002). Entretanto, a pesquisa sobre a influência das partes interessadas na identidade organizacional é emergente, ainda há muito a aprender sobre a natureza 
das relações entre os gestores e os interessados críticos e sobre o papel desses relacionamentos na construção em curso e redefinição de identidade (Barraquier, 2013).

Crane e Ruebottom (2011) propõem uma adaptação para a teoria das partes interessadas em que os interessados são conceituados com base em sua identidade social. Os stakeholders têm sido predominantemente definidos unicamente pela sua função econômica genérica para consumir, investir, fornecer, etc. Tais categorias ignoram a reflexão social, os laços de coesão do grupo, a identidade e a diferença que normalmente formam a base para fazer a reivindicação em relação à empresa. Esse foco enfatiza a necessidade de afastar-se das simplificações oferecidas pela identificação baseada em função de stakeholder padrão, tais como empregado, fornecedor, concorrente, etc., para a identificação das partes interessadas, como indivíduos com identidades e interesses específicos. Handelman (2006) apresenta os constituintes da sociedade não como atores secundários que desempenham um papel subserviente aos primários, baseados em transações econômicas das partes interessadas, mas como poder, contratado, atores sociais culturalmente adaptados que se apresentam às empresas uma gama de interesses sociais e econômicos conflituantes.

Achterkamp e Vos (2007) analisaram as potenciais contribuições de sistemas do pensamento crítico, especialmente a noção crítica de fronteira, para resolver o problema da identificação dos stakeholders. $\mathrm{O}$ que é e quem são incluídos ou excluídos é crucial: a fronteira diferente do sistema pode resultar em uma análise diferente do problema e, consequentemente, em diferentes soluções ou alterações. A essência da crítica de fronteira, portanto, é reconhecer, de forma justificada, a inevitável parcialidade.

Gonçalves, Boaventura, Costa e Fischmann (2008) identificaram os stakeholders através de entrevistas com profissionais do setor hospitalar no estado de São Paulo. Eles forneceram como sugestão, uma lista das partes interessadas para os entrevistados (Tabela 1), com a opção de inclusão de outros.

Tabela 1. Lista sugestiva dos stakeholders do setor hospitalar do estado de São Paulo

\begin{tabular}{|c|c|c|c|}
\hline 1. Indústria Farmacêutica & $\begin{array}{l}\text { 2. Indústria de } \\
\text { Equipamentos } \\
\text { Médicos }\end{array}$ & 3. Indústria de Material Médico-Hospitalar & 4. Saúde Suplementar \\
\hline 5. Instituições de Pesquisa & $\begin{array}{l}\text { 6. Ministério da } \\
\text { Saúde }\end{array}$ & 7. SUS & 8. Médicos \\
\hline 9. Pacientes & 10. Sindicatos & 11. ONGs & $\begin{array}{l}\text { 12. Instituições } \\
\text { Financeiras }\end{array}$ \\
\hline 13. Imprensa & 14. Concorrentes & $\begin{array}{l}\text { 15. Associação dos Hospitais do Estado de São } \\
\text { Paulo (AHESP), Associação Médica Brasileira } \\
\text { (AMB), Associação Nacional Hospitais Privados } \\
\text { (ANAHP), Conselho Regional de Enfermagem } \\
\text { (COREN), etc.) }\end{array}$ & 16. Outros (especificar) \\
\hline
\end{tabular}

Fonte: Adaptado de Gonçalves et al. (2008, p. 93)

Mainardes, Alves, Raposo, e Domingues (2011) ao identificarem os stakeholders das onze Universidades Públicas Portuguesas, optaram por fazê-los de modo empírico, diferentemente - segundo esses acadêmicos - da grande parte dos investigadores, das partes interessadas das universidades, que o fazem a partir da literatura via suposições.

Duggan, Farnsworth, e Kraak (2013), com o objetivo de informar o desenho de uma interface de comunicação multiusuário para a gestão das pescas, identificaram grupos de stakeholders funcionais e investigaram dois métodos contrastantes para categorizar as partes interessadas: a categorização intuitiva 
- que dependeram da interpretação do alocador e julgamento profissional de cada uma das partes interessadas - e a categorização baseada em evidências - onde as informações de interesse das partes interessadas foram recolhidas a partir de uma análise preliminar da literatura dos interessados, publicamente disponíveis, em que apenas as publicações entre 2007-2012 foram incluídas. Embora não sem limitações, concluíram que a categorização baseada em evidências pode levar a uma categorização das partes interessadas coerente e homogênea, o que minimiza os pressupostos sobre os interesses dos stakeholders, pois a confiança na intuição pode ser enganadora.

Schiller, Winters, Hanson, e Ashe (2013) argumentam que as partes interessadas são uma importante fonte de informação na pesquisa em saúde, proporcionando perspetivas críticas e novos insights sobre os complexos determinantes da saúde, e embora um rico corpo de literatura envolva a Teoria dos Stakeholders, um processo sistemático de identificação das partes interessadas de saúde, na prática, não existe. Esses pesquisadores asseveram, ainda, que a interseção de ambientes construídos e sociais com a mobilidade dos idosos é uma área de pesquisa que é fundamentalmente interdisciplinar e se beneficiariam de uma melhor compreensão na perspetiva das partes interessadas.

Para Grandy (2013), no que tange às organizações sem fins lucrativos, para que possam alcançar o sucesso, há uma exigência de que os seus líderes tenham uma compreensão profunda da natureza complexa das relações das partes interessadas. A mensuração desse sucesso precisa ser multidimensional em termos de natureza e que esteja ligado diretamente à missão e ao contexto organizacional, ao invés de estar baseado apenas em medidas genéricas.

Trapp (2014) procurou obter uma maior compreensão das características centrais da influência das partes interessadas na tomada de decisão estratégica de responsabilidade social, para avaliar a medida em que as práticas de gestão dos stakeholders, na realidade, parecem refletir as diversas conceituações ideais de gestão dos interessados apresentados pela teoria. Esta avaliação baseou-se na análise de entrevistas estruturadas em profundidade com gestores de responsabilidade social empresarial de dezasseis líderes da indústria dinamarquesa.

\section{Metodologia}

Este trabalho assume a forma de uma pesquisa descritiva. Um inquérito por questionário foi realizado em Araxá/MG. Foram distribuídos 380 formulários aos funcionários dos hospitais filantrópicos, de origem religiosa, a questionar sobre as suas perceções a respeito dos principais grupos interessados nas atividades da organização hospitalar.

O objetivo aqui foi identificar os stakeholders mais relevantes partindo de uma visão interna, ou seja, uma perspetiva de dentro para fora sobre os interessados na organização. A técnica metodológica utilizada para mensurar a relevância de cada stakeholder, foi a Escala de Diferencial Semântico que, segundo Gil (2009, p. 145) “é uma técnica utilizada para medir o significado atribuído a conceitos, desenvolvida por Osgood, Suci, e Tannenbaum". A escala contempla cinco níveis, desde: 1 - irrelevante; 2 - pouco relevante; 3 - indiferente; 4 - relevante; 5 - muito relevante. Assim, foi apresentada aos respondentes uma lista sugestiva das partes interessadas, definida em pesquisa anterior, realizada no setor hospitalar no estado de São Paulo (Gonçalves et al., 2008). 
Os dados coletados foram depois tratados, apresentados e representados em um diagrama (figura 3) no formato de uma roda, onde o hospital se encontra no centro e os stakeholders nas extremidades dos raios, conforme o modelo preconizado por Freeman (1984). O grau de relevância (de acordo com a Escala de Diferencial Semântico) foi definido com base no maior percentual indicado pelos respondentes de cada item da lista das partes interessadas e representado no diagrama em formatos distintos, sendo que quanto maior a elipse e mais perto do centro, mais relevante é o stakeholder.

As elipses foram ligadas com setas fortes ou fracas, com uma ou duas pontas indicativas, considerando a relação de influência entre cada grupo de interessados e o hospital. Esta relação foi dividida em 6 categorias:

1. Somente influencia o hospital (só uma seta forte direcionada para o hospital);

2. Mais influencia do que é influenciado pelo hospital (duas setas: uma seta forte direcionada ao hospital e outra seta fraca direcionada ao interessado);

3. Influencia e é influenciado pelo hospital de modo equilibrado (só uma seta forte com as duas pontas indicativas);

4. Mais é influenciado do que do que influencia o hospital (duas setas: uma seta forte direcionada ao interessado e outra seta fraca direcionada ao hospital);

5. Somente é influenciado pelo hospital (só uma seta forte direcionada para o interessado);

6. Não influencia e nem é influenciado pelo hospital (só uma reta de ligação sem pontas indicativas); e

7. Não sabe responder.

Para definir a categoria de relação de influência utilizou como critério a categoria com o maior percentual indicado pelos respondentes.

\section{Apresentação dos resultados}

Do total de 380 formulários distribuídos, 117 questionários de retorno, o que equivale a uma taxa de resposta de $31 \%$, pode ser considerado um número interessante. O perfil dos respondentes está demonstrado graficamente na figura 2, onde o destaque ficou para os técnicos em enfermagem com a participação de $31 \%$, seguidos por médicos com $18 \%$, enfermeiros e profissionais do setor administrativo dos hospitais com $16 \%$.

\section{Perfil dos respondentes}

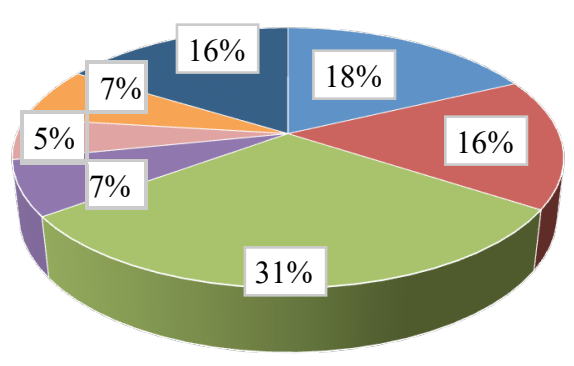
Médicos
Enfermeiros
Técnicos em Enfermagem
- Assistentes de Enfermagem
Fisioterapeutas e Farmacêuticos
- Biólogos e Psicólogos
- Administrativos

Figura 2: Perfil dos respondentes 
O grau de relevância dos stakeholders foi classificado na seguinte ordem: médicos, com o maior percentual (83\%) de muito importante; pacientes, com o segundo maior percentual (81\%) de muito importante; o Sistema Único de Saúde - SUS - com o terceiro maior percentual (65\%) de muito importante; seguidos pela Indústria Farmacêutica (57\%); Indústria de Equipamentos Médicos (56\%); Indústria de Material Médico-Hospitalar (55\%); Ministério da Saúde (46\%); e Instituições de Pesquisa (45\%). O stakholder Saúde Suplementar teve o maior percentual de importante (55\%) seguido das Associações (43\%), Instituições Financeiras (37\%), Concorrentes (32\%), ONGs (31\%), Imprensa (28\%) e Sindicatos (27\%). Esses dados resultaram na composição do diagrama (figura 3) em termos de tamanho e proximidade da elipse (Stakeholder) em relação ao centro (Hospital).

A relação de influência com maior percentual em destaque, segundo os respondentes, foram apenas duas categorias assim distribuídas:

- Mais influencia do que é influenciado pelo hospital: Ministério da Saúde (29\%), SUS (26\%) e Sindicatos $(21 \%)$;

- Influencia e é influenciado pelo hospital de modo equilibrado: Indústria de Material MédicoHospitalar (56\%), Indústria de Equipamentos Médicos (49\%), Indústria Farmacêutica (44\%), Saúde Suplementar (44\%), Médicos (38\%), Pacientes (38\%), Associações (38\%), ONGs (27\%), Concorrentes (32\%), Instituições de Pesquisa (25\%), Imprensa (24\%) e Instituições Financeiras $(21 \%)$

Os resultados possibilitaram a construção de um diagrama representativo apresentando o mapa dos stakeholders (figura 3).

Os grupos interessados que os respondentes tiveram maior dificuldade para identificar a relação de influência com o hospital foram a imprensa, os concorrentes, as associações e as instituições financeiras, com a indicação de $30 \%, 26 \%, 24 \%$ e $23 \%$, respetivamente, da opção não sabe responder.



Figura 3. Diagrama - Mapa de Stakeholders 


\section{Discussão}

Os dados mostram que, internamente, os médicos, os pacientes e o "Sistema Único de Saúde" são percebidos como os interessados mais relevantes. Contrariamente ao esperado, o grau de relevância dos beneméritos doadores de contribuidores financeiros é marginal, aliás, praticamente ignorada pelos participantes. No formulário de inquérito havia um espaço para a sugestão de outros interessados, onde esperávamos a inclusão, por parte dos participantes, de empresas colaboradoras, governos municipais das cidades vizinhas, comunidades religiosas e instituições de classes, como exemplos. Assim, os resultados obtidos são vistos com surpresa uma vez que esses hospitais são dependentes de contribuições financeiras voluntárias, não só em termos de sustentabilidade financeira, mas principalmente porque essas contribuições são um indicador direto da sua legitimação social.

Foram consideradas 6 categorias para retratar a relação de influência entre cada grupo de interessados e o hospital. Entretanto, apenas duas se destacaram com um maior percentual de indicação por parte dos respondentes. A primeira categoria com destaque foi a mais influencia do que é influenciado pelo hospital, representada no mapa com uma seta forte direcionada para o hospital e outra fraca direcionada aos seguintes interessados: Ministério da Saúde (29\%), SUS (26\%) e Sindicatos (21\%). A segunda categoria com destaque foi influencia e é influenciado pelo hospital de modo equilibrado, representada no mapa com uma seta forte com as duas extremidades direcionadas, uma para o hospital e outra para os seguintes grupos de interessados: Indústria de Material Médico-Hospitalar (56\%), Indústria de Equipamentos Médicos (49\%), Indústria Farmacêutica (44\%), Saúde Suplementar (44\%), Médicos (38\%), Pacientes (38\%), Associações (38\%), ONGs (27\%), Concorrentes (32\%), Instituições de Pesquisa (25\%), Imprensa (24\%) e Instituições Financeiras (21\%).

Outra constatação foi a que 17\%, em média, dos respondentes tiveram dificuldade em identificar a categoria que retrata a influência entre os interessados e a instituição hospitalar. Cabe destacar quatro grupos de interessados, cuja dificuldade de identificação desse item foi muito acima da média: a imprensa, os concorrentes, as associações e as instituições financeiras, com a indicação de 30\%, 29\%, $25 \%$ e $23 \%$ respetivamente, da opção não sabe responder. Nos parece relevante a influência da imprensa sobre o hospital, especialmente através da informação gerada à sociedade. No entanto, não foi a realidade constatada através dos números da pesquisa. Além disso, cabe ainda ressaltar mais uma vez a ausência de doadores que parece-nos influenciar diretamente esse tipo de hospital, cuja característica principal está no fato de ser filantrópicos. Sem falar do caráter religioso de origem dessas instituições de saúde, o que seria natural ser constatado uma relação direta de influência das comunidades religiosas sobre elas, e que nem mesmo foram sugeridas pelos respondentes.

Esta pesquisa baseia-se em uma amostra que não pode ser considerada representativa e, portanto, qualquer generalização das conclusões deve ser feita com muito cuidado. Além disso, ela se concentra apenas nas perceções dos trabalhadores e, portanto, traduz apenas perspetiva de uma das partes interessadas, sendo necessário muito mais para obter uma matriz completa e efetivamente representativa dos grupos interessados nos hospitais. No entanto, este trabalho fornece novos insights sobre a identificação dos grupos de partes interessadas no que diz respeito a hospitais filantrópicos, de origem religiosa, abrindo assim espaço para a discussão sobre a importância e as especificidades da gestão destas 
relações dos hospitais filantrópicos e religiosos com os médicos, os pacientes e o SUS. Cria-se, assim, um ambiente motivacional para um estudo mais robusto e de profundidade, com uma amostra de maior amplitude a envolver a opinião de outros importantes grupos interessados nas instituições hospitalares.

\section{Referências}

Aaltonen, K., Jaakko, K., \& Tuomas, O. (2008). Stakeholder salience in global projects. International Journal of Project Management, 26(5), 509-516. doi:10.1016/j.ijproman.2008.05.004

Achterkamp, M. C., \& Vos, J. F. J. (2007). Critically identifying stakeholders. Systems Research and Behavioral Science, 24(1), 3-14. doi:10.1002/sres.760

Ackermann, F., \& Eden, C. (2011). Strategic Management of Stakeholders: Theory and Practice. Long Range Planning, 44(3), 179-196. doi:10.1016/j.lrp.2010.08.001

Avetisyan, E., \& Ferrary, M. (2013). Dynamics of Stakeholders' Implications in the Institutionalization of the CSR Field in France and in the United States. Journal of Business Ethics, 115(1), 115-133. doi:10.1007/s10551-012-1386-3

Barraquier, A. (2013). A Group Identity Analysis of Organizations and Their Stakeholders: Porosity of Identity and Mobility of Attributes. Journal of Business Ethics, 115(1), 45-62. doi:10.1007/s10551-012-1363-x

Brugha, R., \& Varvasovszky, Z. (2000). Stakeholder analysis: a review. Health Policy and Planning, 15(3), 239-246.

Bryson, J. M. (2004). What to do when Stakeholders matter. Public Management Review, 6(1), 21-53. doi:10.1080/14719030410001675722

Crane, A., \& Ruebottom, T. (2011). Stakeholder Theory and Social Identity: Rethinking Stakeholder Identification. Journal of Business Ethics, 102(1), 77-87. doi:10.1007/s10551-011-1191-4

Duggan, D. E., Farnsworth, K. D., \& Kraak, S. B. M. (2013). Identifying functional stakeholder clusters to maximise communication for the ecosystem approach to fisheries management. Marine Policy, 42, 56-67. doi:10.1016/j.marpol.2013.01.023

Fassin, Y. (2009). The Stakeholder Model Refined. Journal of Business Ethics, 84(1), 113-135. doi:10.1007/s10551-008-9677-4

Freeman, R. E. (1984). Strategic Management: A Stakeholder Approach. Pitman.

Friedman, A. L., \& Miles, S. (2006). Stakeholders!: Theory and Practice: Theory and Practice. Oxford University Press.

Frooman, J. (1999). Stakeholder Influence Strategies. The Academy of Management Review, 24(2), 191205. doi: $10.2307 / 259074$

Gil, A. C. (2009). Como elaborar projetos de pesquisa (4ª ed.). São Paulo: Atlas. 
Gonçalves, P. C., Boaventura, J. M. G., Costa, B. K., \& Fischmann, A. A. (2008). Stakeholders na atividade hospitalar: uma Investigação setorial no Estado de São Paulo. Revista de Administração FACES Journal, 7(2), 84-101.

Grandy, G. (2013). An exploratory study of strategic leadership in churches. Leadership \& Organization Development Journal, 34(7), 616-638. doi:10.1108/LODJ-08-2011-0081

Handelman, J. M. (2006). Corporate identity and the societal constituent. Journal of the Academy of Marketing Science, 34(2), 107-114. doi:10.1177/0092070305284970

Kaler, J. (2002). Morality and Strategy in Stakeholder identification. Journal of Business Ethics, 39(1-2), 91-100. doi:10.1023/A:1016336118528

Lafreniere, K. C., Deshpande, S., Bjornlund, H., \& Hunter, M. G. (2013). Extending stakeholder theory to promote resource management initiatives to key stakeholders: a case study of water transfers in Alberta, Canada. Journal of Environmental Management, 129, 81-91. doi:10.1016/j.jenvman.2013.06.046

Mahoney, J. T. (2012). Towards a stakeholder theory of strategic management. Em J. M. R. Martí \& J. E. R. Costa (Eds.), Towards a New Theory of the Firm: Humanizing the Firm and the Management Profession (pp. 153-182). Bilbao: Fundacion BBVA.

Mainardes, E. W. (2010). Gestão de Universidades Baseada no Relacionamento com os seus Stakeholders (Dissertação de Doutorado em Administração). Universidade da Beira Interior, Covilhã, portugal.

Mainardes, E. W., Alves, H., Raposo, M., \& Domingues, M. J. (2011). Categorização Por Importância Dos Stakeholders Das Universidades. Revista Ibero-Americana de Estratégia, 9(3), 04-43. doi:10.5585/riae.v9i3.1681

Mitchell, R. K., Agle, B. R., \& Wood, D. J. (1997). Toward a Theory of Stakeholder Identification and Salience: Defining the Principle of Who and What Really Counts. The Academy of Management Review, 22(4), 853-886. doi:10.2307/259247

Neville, B. A., \& Menguc, B. (2006). Stakeholder Multiplicity: Toward an Understanding of the Interactions between Stakeholders. Journal of Business Ethics, 66(4), 377-391. doi:10.1007/s10551-006-0015-4

Olander, S., \& Landin, A. (2005). Evaluation of stakeholder influence in the implementation of construction projects. International Journal of Project Management, 23(4), 321-328. doi:10.1016/j.ijproman.2005.02.002

Parent, M. M., \& Deephouse, D. L. (2007). A Case Study of Stakeholder Identification and Prioritization by Managers. Journal of Business Ethics, 75(1), 1-23. doi:10.1007/s10551-007-9533-y

Schiller, C., Winters, M., Hanson, H. M., \& Ashe, M. C. (2013). A framework for stakeholder identification in concept mapping and health research: a novel process and its application to 
older adult mobility and the built environment. BMC Public Health, 13(1), 1-9.

doi:10.1186/1471-2458-13-428

Trapp, N. L. (2014). Stakeholder involvement in CSR strategy-making? Clues from sixteen Danish companies. Public Relations Review, 40(1), 42-49. doi:10.1016/j.pubrev.2013.11.005

Tullberg, J. (2013). Stakeholder theory: Some revisionist suggestions. The Journal of Socio-Economics, 42, 127-135. doi:10.1016/j.socec.2012.11.014

Vos, J. F. J. (2003). Corporate social responsibility and the identification of stakeholders. Corporate Social Responsibility and Environmental Management, 10(3), 141-152. doi:10.1002/csr.39 